\title{
LHC Expectations (Machine, Detectors and Physics)
}

\author{
Günther Dissertori* \\ ETH Zürich, Switzerland \\ E-mail: guenther.dissertoridcern.ch
}

Starting in two years from now, particle physics will enter a new regime in terms of energies and luminosities, thanks to the Large Hadron Collider (LHC) at CERN. This report summarizes the status of the preparations, both for the machine and the detectors, as of fall 2005. The commissioning and start-up scenarios are outlined and some highlights from the very rich physics programme are given, concentrating on measurements of Standard Model processes, as well as on early discovery scenarios. The prospects of B-physics and heavy ion collisions at LHC are also briefly discussed. The report concludes with an outlook on the ultimate physics reach and on upgrade scenarios.

International Europhysics Conference on High Energy Physics

July 21st - 27th 2005

Lisboa, Portugal

${ }^{*}$ Speaker. 


\section{Introduction}

We are approaching the start-up of the world's most powerful particle accelerator ever built. In about two years from now, CERN's Large Hadron Collider (LHC) [1] will starts its operation. Thanks to the unprecedented energies and luminosities, it will give particle physicists the possibility to explore the $\mathrm{TeV}$ energy range for the first time and hopefully discover new phenomena, which go beyond the so successful Standard Model (SM).

The physics motivations for this new endeavour are manifold. Above all, it is believed that the origin of electro-weak symmetry breaking will be elucidated. Concretely speaking, this might consist in the discovery of one or more Higgs bosons and thus confirm the prediction that there is spontaneous symmetry breaking via the Higgs mechanism [2]. On the other hand, if no evidence is obtained for a Higgs mechanism, we nevertheless expect new phenomena to show up in the $\mathrm{TeV}$ energy range, which after all have to ensure the conservation of unitarity. The latter is known to be violated, for example in the scattering of the longitudinal components of two $\mathrm{W}$ bosons, if no new phenomena set in at the $\mathrm{TeV}$ scale.

The other main field of activity will be the search for new types of symmetries and particles, most notably Supersymmetry (SUSY). We refer to [3] for an overview of SUSY and its phenomenological implications. SUSY is the most prominent and carefully studied model of all proposed extensions of the SM. It postulates a symmetry between fermions and bosons and introduces a rich new spectrum of particles. This theory has several strong motivations. For example, it proposes a rather natural solution of the hierarchy problem, if supersymmetric partners of the SM particles appear with masses below or around the TeV scale. This would prevent the Higgs mass to acquire enormously large radiative corrections and eliminate the need to have an unnatural fine tuning in order to explain the apparently small Higgs mass. The appearance of SUSY particles would also lead to the convergence of the electro-weak and strong coupling constants at an energy of about $10^{16} \mathrm{GeV}$, as generally foreseen in scenarios of Grand Unified Theories (GUT). Finally, some implementations of SUSY provide an excellent candidate for the dark matter observed in our Universe, namely the weakly interacting and stable lightest neutralino.

Recently other solutions for the hierarchy problem have been put forward, which postulate the existence of Extra Dimensions (ED). Some of these models [4] conjecture that the fundamental scale of gravity could be as low as the TeV scale. The Planck scale only appears as a derived scale because of the large volume of the EDs and the fact that only gravity can propagate there, whereas all SM fields are confined to a four-dimensional brane. Thus we see only a small part of the total gravitational flux, which explains the relative weakness of gravity compared to the other SM interactions. Other models exist [5] which try to explain the hierarchy problem by a very strong curvature of the EDs. In general, the phenomenology of EDs foresees the production of gravitons via parton scattering at the LHC. These gravitons could escape into the EDs, leading to an apparent violation of energy-momentum conservation in our four-dimensional world, or decay to SM particles in a resonant-like behaviour.

Of course, at LHC we will also search for new interactions and their related carrier particles, such as new vector bosons ( $\left.\mathrm{Z}^{\prime}, \mathrm{W}^{\prime}\right)$ with masses of a few $\mathrm{TeV} / \mathrm{c}^{2}$. These particles arise in models which extend the gauge group of the SM, as for example the recent Little Higgs models (see eg. [6] and references therein). Another proposal to explain electro-weak symmetry breaking is given 
by Technicolor models [7]. Also there we would expect the appearance of heavy resonances.

Besides the direct searches for physics beyond the SM, precision studies of the heavy flavour sector could lead to indirect evidence for new physics, for example via an enhancement of otherwise very rare decays. In particular, the very copious production of $\mathrm{B}_{\mathrm{s}}$ mesons will allow to complement the measurements at the B-factories currently in operation. The flavour mixing parameters which appear in the Cabbibo-Kobayashi-Maskawa (CKM) matrix will be measured using several different decay channels, hopefully leading to a better understanding of CP-violation in the B-sector.

Finally, the LHC will also allow to collide heavy ions. The unprecedented energy densities achieved in these collisions are expected to lead to the formation of new forms of partonic matter, most notably a quark-gluon plasma. The properties of this new state of matter, as well as the phase transition to hadronic matter will be the subject of an intense research.

This very rich physics programme will be pursued at CERN by observing proton-proton collisions (as well as heavy ion collisions) at four experimental sites around the LHC ring. It will be installed in the former LEP tunnel, about 100 m underground with a circumference of approximately $27 \mathrm{~km}$. Two of the four experiments, ATLAS and CMS, will be large general purpose detectors designed to cover practically the whole range of physics questions outlined above. The other two experiments are optimized for the study of B-physics ( $\mathrm{LHCb}$ ) and heavy ion collisions (ALICE).

In the following we will summarize the status of the preparations of the machine and the detectors and describe the planned start-up scenarios. In a more detailed discussion of the foreseen physics analyses, emphasis will be given to the early physics reach. The report is concluded with an outlook on the later physics reach and possible LHC upgrade scenarios. Other reviews of physics at the LHC and its preparations can be found in Refs. [8], [9] and [10].

\section{Construction status}

\subsection{Status of the LHC construction}

The LHC will be a proton-proton collider with an energy per beam of $7 \mathrm{TeV}$, a factor of seven larger than the currently highest energy achieved in the world, namely with the TEVATRON at FERMILAB. Its main components will be 1232 superconducting dipoles, each $14.2 \mathrm{~m}$ long (magnetic length). When operated at their nominal temperature of $1.9 \mathrm{~K}$, a magnetic field of $8.33 \mathrm{~T}$ is achieved. They are of the " 2 in 1 " type, meaning that the apertures $(56 \mathrm{~mm})$ for both beams have a common mechanical structure and cryostat. The cryogenic services line (QRL), which distributes and supplies the liquid helium to the magnets, will be installed next to the beam line.

In total 2808 bunches with a nominal intensity of $1.1 \times 10^{11}$ protons/bunch and a bunchspacing of $24.95 \mathrm{~ns}$ will circulate in the ring, leading to a nominal luminosity of $10^{34} \mathrm{~cm}^{-2} \mathrm{~s}^{-1}$. The total energy stored in the beam will reach a macroscopic value of $350 \mathrm{MJ}$, which imposes severe constraints and requirements on its safe operation, since an uncontrolled beam loss unavoidably would damage the equipment. It is worth noting that in terms of stored energy per beam, the LHC exceeds all previous and existing machines by a factor of 200 , thus we enter unexplored territory, indeed.

The layout of the machine consists of eight independent sectors, in order to handle in a distributed manner the total energy of $10 \mathrm{GJ}$ stored in the magnets. Being the first machine with 

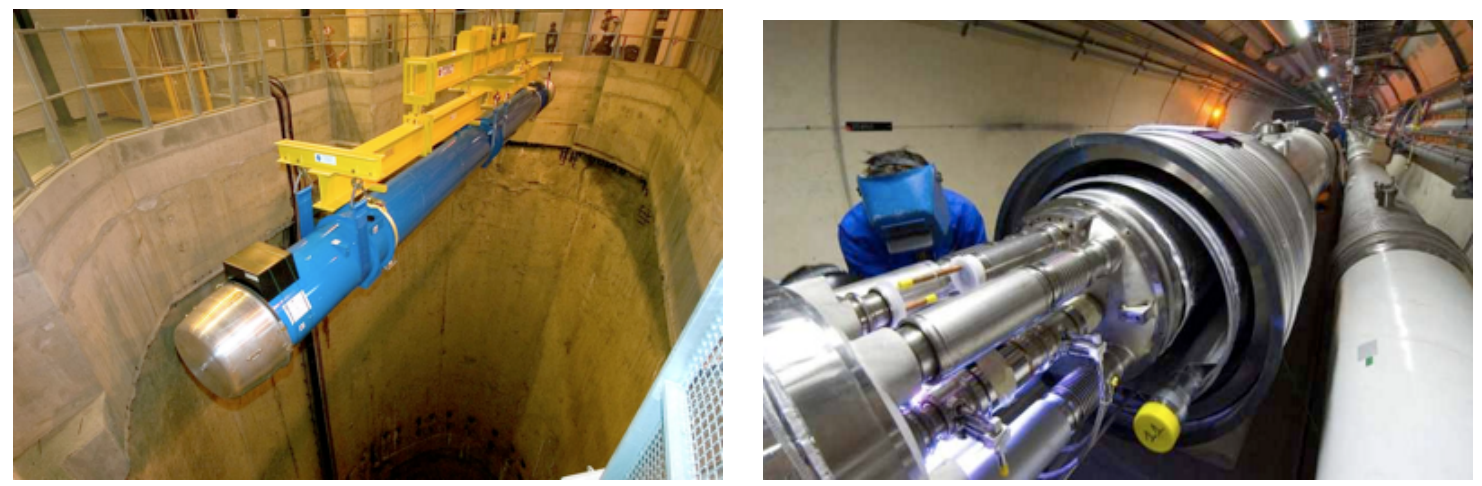

Figure 1: Left : Lowering of the first superconducting LHC dipole into the tunnel, March 2005. Right : Interconnection of two dipoles, next to the cryogenic services line.

independent sectors, it presents an enormous challenge for its control and powering. Besides the four interaction points, where the beams will cross at a nominal angle of $285 \mu \mathrm{rad}$, there are warm insertion regions for beam dump, cleaning and acceleration.

The magnet production proceeds very well and is on schedule. To date more than 800 magnets of excellent quality have been delivered. The first superconducting dipole was lowered into the accelerator tunnel on Monday, $7^{\text {th }}$ March (Fig. 1), and by now about 120 dipoles are already installed in the tunnel. Prior to the magnets the cryogenic services line has to be installed. This has caused problems in the past, to which CERN has reacted promptly and successfully implemented a recovery plan. To date QRL components for four of the eight LHC sectors have been delivered. The installation proceeds in three sectors in parallel and almost two sectors have been fully equipped. Recently the first QRL sub-sector has been successfully cooled down, after solving some minor problems which appeared during the pressure tests.

The installation of the LHC in the tunnel is on the critical path for the first collisions. The LHC schedule [11] foresees a parallel installation of pairs of sectors, the last of them to be completed in June 2007. The first sector pair (sectors 7 and 8) shall be completed by May 2006 and cooled down for a first test with beam. This will involve beam injection from the SPS down the TI8 transfer line, right off point 8 . The beam will pass through interaction point $8(\mathrm{LHCb})$ and then through sector 8-7 before reaching a temporary beam dump. This will be an important system test and allow to pre-commission essential data acquisition and correction procedures.

\subsection{Status of the experiments}

The largest of the four detectors, ATLAS [12], is currently being installed in the experimental cavern. Its enormous size ( $25 \mathrm{~m}$ diameter, $46 \mathrm{~m}$ total length and 7000 tons overall weight) is determined by the muon system, based on air-core toroids equipped with muon chambers. At the end of summer 2005 all eight of the barrel toroid coils have been installed (Fig. 2, left), and the construction and installation of the muon chambers proceeds on schedule. The construction of the electromagnetic calorimeter, a lead/liquid argon sampling calorimeter with accordion design, is completed. It shares the cryostat with the superconducting solenoid surrounding the central tracking system. After a successful cold test the cryostat has been lowered into the cavern in 
October 2004. Also the hadronic tile calorimeter is already underground and has registered first cosmic rays in June 2005. The planning for the construction of the tracker, consisting of a transition radiation detector, silicon strip and pixel detectors, is tight. The emphasis is now switching to the integration, final installation and commissioning of the sub-systems. An important step in this direction was a combined test beam campaign in fall 2004, where parts of all sub-detectors have been integrated and read out together, based on a common data acquisition and detector control system. The six-months running period has led to a good global operation experience. The common ATLAS software is now employed to analyse a data set of about 4.5 TByte.
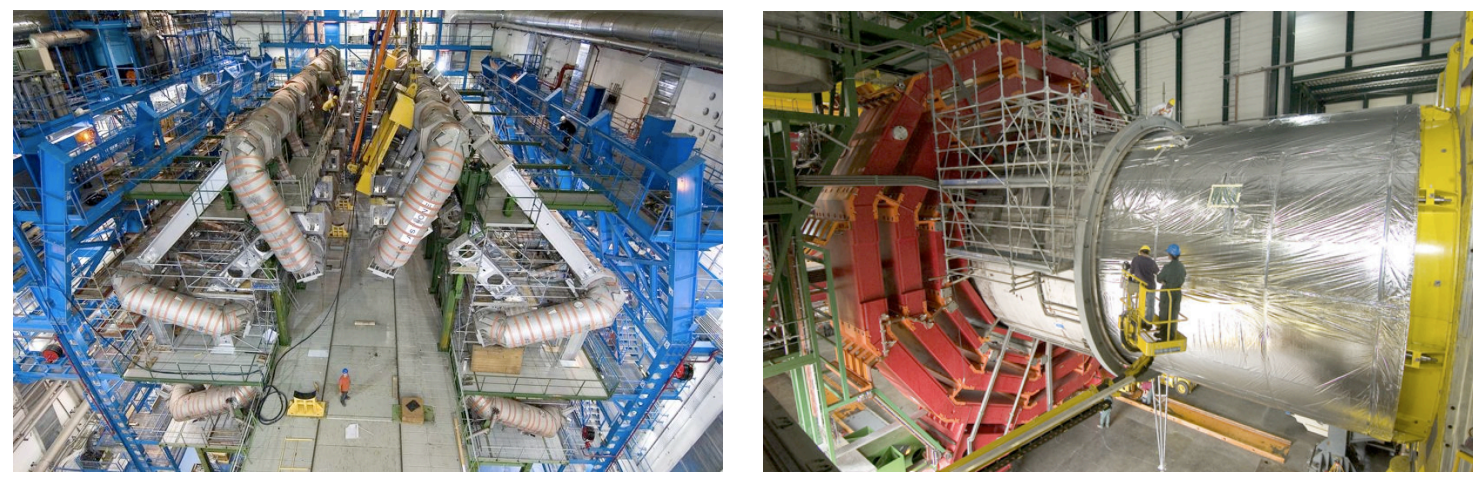

Figure 2: Left : All of the eight ATLAS barrel toroid coils installed in the cavern. Right : Insertion of the CMS solenoid into the outer vacuum tank.

The second of the two general purpose detectors, CMS [13], is indeed compact when compared to ATLAS. Its overall diameter is $15 \mathrm{~m}$, its length $21.6 \mathrm{~m}$ and its total weight 12500 tons. The main design difference is that it has only one magnet system. The central superconducting solenoid, with an inner diameter of $6.32 \mathrm{~m}$ and a magnetic field of $4 \mathrm{~T}$, is large enough to house the tracking system, the electromagnetic and the hadronic calorimeters. The magnetic return yoke, made of iron rings and disks, is instrumented with muon chambers. The overall assembly of the big mechanical parts takes place in a surface hall above the experimental cavern at point 5 of LHC. A major milestone for the magnet has been achieved in summer 2005 with the final assembly of the five coil modules, its swivelling and insertion into the outer vacuum tank (Fig. 2, right), held by the central barrel wheel. The inner vacuum tank has been inserted in November 2005. The next important steps will be the first cool-down, followed by the magnet test in spring 2006. This will be combined with a $20^{\circ}$ slice test, where parts of all sub-detectors will be read out on cosmic ray triggers. This will be the first trial of the CMS operation procedures. The lowering of the heavy elements into the cavern will start immediately after. Concerning the sub-detectors, the muon chamber installation is progressing very well and the hadron calorimeter, a brass/scintillating tiles sampling calorimeter, is completed. The production of the 76848 lead-tungstate crystals for the electromagnetic calorimeter (ECAL) is on the critical path. Its end-caps will only be installed during the first long shutdown at the beginning of 2008. The ECAL electronics integration is a well established procedure and test beam measurements in 2004 have shown excellent performance in terms of energy resolution and noise. Finally, the CMS silicon tracker will be the largest ever built, comprising about $250 \mathrm{~m}^{2}$ of silicon sensors. The sensor module production will be completed in spring 2006. The complete 
tracker has to be integrated at CERN by November 2006 in order to be in line with the overall installation schedule. Similar to the ECAL endcaps, the pixel detector, although ready, will only be installed after the pilot run in winter 2007-2008.

Next to CMS the TOTEM experiment [14] will be installed. It consists of CSC and GEM telescopes in the very forward direction around the beam pipe, covering pseudo-rapidity ranges from 3.1 to 6.5 , as well as of roman pots up to a distance of $220 \mathrm{~m}$ from the CMS interaction point. The main goals of this experiment are the measurements of the total, elastic and diffractive proton-proton cross sections, of the particle and energy flow in the very forward direction and an absolute luminosity determination. However, in order to carry out this physics programme, very special LHC running conditions (optics) are required.

Since the b-quark production cross section peaks at the very forward direction, the $\mathrm{LHCb}$ [15] experiment is designed as a single-arm spectrometer, covering a pseudo-rapidity range of $1.8<$ $\eta<4$.9. The magnet, the electromagnetic and hadronic calorimeters, as well as the iron for the muon filters have already been installed in the former DELPHI cavern. Good achievement has been made for the construction of many sub-systems, such as the vertex locator, the tracking chambers and the RICH detectors. LHCb will collect data at a lower luminosity of $2 \times 10^{32} \mathrm{~cm}^{-2} \mathrm{~s}^{-1}$. One of its essential elements, the trigger system, has been re-organized recently, with a final storage rate of $2000 \mathrm{~Hz}$ after the higher level trigger stage, leading to $10^{9}-10^{10} \mathrm{~B}$-hadrons per year.

Finally, the ALICE [16] detector is designed to fully exploit the heavy ion operation of LHC (lead ions with $2.76 \mathrm{TeV} /$ nucleon and an initial luminosity of $10^{27} \mathrm{~cm}^{-2} \mathrm{~s}^{-1}$ ). It is installed in the former L3 cavern and re-uses the L3 magnet. Its main element is a huge time projection chamber of $88 \mathrm{~m}^{3}$, which will allow the reconstruction of several thousand tracks per unit of rapidity. Further emphasis is given to particle identification. Dedicated sub-systems cover restricted angular regions, such as a forward muon spectrometer based on an additional dipole magnet. An almost complete detector will be ready for the first proton-proton collisions in 2007. The full detector system will be operational for the first heavy ion run foreseen in 2008 .

\section{Commissioning and start-up scenarios}

\subsection{LHC commissioning and early operations}

In order to understand the various steps in the start-up of the LHC operations, it is instructive to shortly review the main machine parameters and the corresponding limitations. The beam energy of $7 \mathrm{TeV}$ is limited by the maximal magnetic dipole field available and its field quality. This, on the other hand, is determined by the industrially available magnet technology. The chosen LHC magnet parameters (magnetic field, maximal sustainable current density, temperature) leave small margins for thermal and mechanical stresses, which ultimately lead to quenches.

The nominal bunch intensity (protons/bunch) is $N=1.15 \times 10^{11}$, with an upper limit of $N=1.7 \times 10^{11}$. With 2808 bunches, this corresponds to a maximal beam current of $I=0.85 \mathrm{~A}$. The limits on these parameters are imposed by several effects. Beam-beam effects, which lead to a tune $(Q)$ spread, have to be minimized in order to avoid resonances, since the allowed region in $Q$-space is rather restricted. Resonances are also avoided by an excellent magnetic field quality, by correction circuits and optimal feedback from the beam instrumentation. A high operation efficiency and thus the maximal achievable integrated luminosity is obtained by minimizing quenches 
and beam aborts. Here collimators and the cleaning insertions play an important role. However, the sustainable radiation dose in the cleaning insertions is just compatible with the nominal beam intensities. Also the enormous energy stored in the beams represents a serious damage potential and therefore has to be well understood and controlled. The high beam intensities, coupled to badly conducting collimator materials (eg. graphite) induce large wake fields which cause collective beam instabilities. This effect can be controlled by first limiting the beam intensity, and second by a proper choice of collimators. In a first phase, graphite collimators will be used, thanks to their robustness and thus increased machine safety. Since with this choice the beam current is limited to $I<0.3 \mathrm{~A}$, in a second phase they will be replaced by copper collimators, allowing for $I<0.85 \mathrm{~A}$. These are good conductors, but would be seriously damaged in case of a full beam impact. Finally, the electron cloud effect [17] puts heat load on the beam screen, which increases for smaller bunch spacing. Recent studies show that the nominal number of bunches allows for a stable operation, if there is a prior conditioning of the surfaces by a so-called beam scrubbing. Initially this effect can be more easily controlled by an increased bunch spacing, for example $75 \mathrm{~ns}$.

The physical beam size, $\sigma=\sqrt{\beta \varepsilon}$, is determined by the machine's $\beta$-function and the emittance $\varepsilon=\varepsilon_{\mathrm{n}} / \gamma$, where $\gamma$ is the Lorentz factor and $\varepsilon_{\mathrm{n}}$ the so-called normalized emittance. The latter is limited to $\varepsilon_{\mathrm{n}}<3.75 \mu \mathrm{m}$ by the injector chain and the main dipole aperture. The luminosity at the interaction points (IPs) increases with smaller beam size $\sigma^{*}$, thus a smaller $\beta$-function. Its value at an IP is denominated $\beta^{*}$. A limit on this parameter $\left(\beta^{*}>0.55 \mathrm{~m}\right)$ is imposed by the physical aperture of the first triplet of superconducting quadrupole magnets around the IPs. The $\beta$-function in proximity of an IP can be approximated by $\beta(s) \approx \beta^{*}+s^{2} / \beta^{*}, s$ being the distance from the IP. Taking a nominal $\sigma^{*}=16.6 \mu \mathrm{m}$ and a distance to the first quadrupoles of $\approx 23 \mathrm{~m}$, the beam size at the quadrupoles is of the order of a millimetre, which starts to be of a similar order of magnitude as the quadrupole aperture.

For nominal bunch intensities and spacing the beam-beam effects near an IP can be reduced by introducing a finite beam crossing angle, $\approx 300 \mu \mathrm{rad}$. This crossing angle is again limited by the aperture of the nearest quadrupole magnets and the corresponding stress on these magnets and the collimators.

All the above mentioned issues have to be taken into account in order to achieve the main objective of the early LHC operation, which is to establish colliding beams as quickly as possible, safely and without compromising further progress. The general approach will be to initially take two multi-bunch beams with moderate intensity to high energy and collide them at zero crossing angle. Graphite collimators will be installed at the beginning.

Currently the hardware commissioning, system tests, machine and transfer lines checkout are foreseen for late spring to early summer 2007. Then the commissioning with beam will start, followed by a pilot run in fall 2007. The start-up of the machine is planned in four stages, approaching gradually the ultimate machine parameters. In a first stage the LHC will run with $43 \times 43$ bunches, moving to $156 \times 156$ bunches with moderate intensities $\left(N \approx 3 \times 10^{10}\right)$, zero to partial squeeze and zero crossing angle. In a second stage with $936 \times 936$ bunches and partial squeeze a luminosity of up to $4 \times 10^{32} \mathrm{~cm}^{-2} \mathrm{~s}^{-1}$ should be reached. The third stage would correspond to the start of the 25 ns operations, with intensities up to $N=5 \times 10^{10}$ and almost full squeeze. During this stage a luminosity of $2 \times 10^{33} \mathrm{~cm}^{-2} \mathrm{~s}^{-1}$ will be approached, which currently is also assumed in the physics studies for the first year(s) of LHC operation. The fourth stage, when all parameters will be pushed 
to the nominal values (luminosity of $10^{34} \mathrm{~cm}^{-2} \mathrm{~s}^{-1}$ ), will only be reached after a few years. It necessitates the exchange of the graphite with copper collimators and the installation of the complete beam dump system. Since it is not clear how fast the first operation stages will be passed through and what the machine operation and detector efficiencies will be, it is very difficult to give a precise estimate of the integrated luminosity on tape at the end of 2008, after the first long physics run. Based on the numbers above, this could be anywhere in the range of 0.1 to $10 \mathrm{fb}^{-1}$ [18].

\subsection{Commissioning of the experiments}

All experiments are expected to be ready by end of June 2007. However, parts or all of the installed sub-detectors can be commissioned and pre-calibrated already well before we have first collisions in LHC. Cosmic ray muons will be used by all detectors in order to obtain initial alignment and calibration constants for the barrel parts mainly. These muons are also very useful for debugging and mapping of dead-channels. An estimate of the rate is approximately $1-5 \mathrm{kHz}$ for muons with an energy at the surface exceeding $10 \mathrm{GeV}$. Out of these a few $\mathrm{Hz}$ might be useful for calibration. It is worth repeating that some sub-detector systems have already been commissioned with cosmic muons in 2005, and CMS foresees a full system test at the surface, with the magnetic field on, in 2006.

Once at least one beam is circulating, beam-halo muons will traverse the experiments and thus allow for further alignment and calibration efforts, now with emphasis on the end-caps of the detectors. The rate for muons with an energy above $100 \mathrm{GeV}$ is estimated to about $1 \mathrm{kHz}$. At the same time, beam-gas events will be registered, which already resemble the later proton-proton collisions. However, the spectrum of the produced tracks is much softer, with typically $p_{\mathrm{T}}<2$ $\mathrm{GeV} / c$. Nevertheless, with a rate of $25 \mathrm{~Hz}$ for reconstructed tracks with $p_{\mathrm{T}}>1 \mathrm{GeV} / c$, coming from a vertex with $|z|<20 \mathrm{~cm}$, it might be possible to obtain a first alignment of the inner trackers to about $100 \mu \mathrm{m}$.

Finally, with the first collisions in hand, the trigger and data acquisition systems will be timedin, the data coherence checked, sub-systems synchronized and reconstruction algorithms debugged and calibrated. The electromagnetic and hadronic calorimeters will be calibrated with first physics events. For example, the initial crystal inter-calibration precision of about $4 \%$ for the CMS ECAL will be improved to about $2 \%$ by using the $\phi$-symmetry of the energy deposition in minimum-bias and jet events. Later the ultimate precision $(\approx 0.5 \%)$ and the absolute calibration will be obtained using $\mathrm{Z} \rightarrow \mathrm{e}^{+} \mathrm{e}^{-}$decays and the $E / p$ measurements for isolated electrons, such as in $\mathrm{W} \rightarrow \mathrm{e} v$ decays [19]. The latter requires a well understood tracking system. The uniformity of the hadronic calorimeters can be checked with single pions and QCD jets. In order to obtain the jet energy scale to a few per-cent or better, physics processes such as $\mathrm{Z}(\rightarrow \ell \ell)+$ jet or $\mathrm{W} \rightarrow 2$ jets in top pair events will be analyzed. Finally, the tracker and muon system alignment will be carried out with generic tracks, isolated muons or $\mathrm{Z} \rightarrow \mu^{+} \mu^{-}$decays. Regarding all these calibration and alignment efforts, the ultimate statistical precision should be achieved after a few days of operation in most cases. Then systematic effects have to be faced, which, eg., implies that pushing the tracker $R \phi$ alignment from an initial $100 \mu \mathrm{m}$ to about $5 \mu \mathrm{m}$ might involve at least one year of data taking. A more detailed review of the initial detectors and their performance can be found in Ref. [18]. 


\section{Early Physics}

The very early goals to be pursued by the experiments, once the first data are on tape, are three-fold : (a) It will be of utmost importance to commission and calibrate the detectors in situ, with physics processes as outline above. The trigger performance has to be understood in a possibly unbiased manner, by analyzing the trigger rates of minimum-bias events, QCD jet events for various thresholds, single and di-lepton as well as single and di-photon events. (b) It will be necessary to measure the main SM processes (cf. section 4.1) and (c) prepare the road for possible discoveries (section 4.2).

It is instructive to recall the event statistics collected for different types of processes. For an integrated luminosity of $10 \mathrm{fb}^{-1}$ per experiment, we expect about $10^{8} \mathrm{~W} \rightarrow \mathrm{e} v$ events on tape, a factor of ten less $\mathrm{Z} \rightarrow \mathrm{e}^{+} \mathrm{e}^{-}$and some $10^{6} \mathrm{t} \mathrm{t} \rightarrow \mu+X$ events. Even if a trigger bandwidth of only $10 \%$ is assumed for QCD jets with $p_{\mathrm{T}}>150 \mathrm{GeV} / c, \mathrm{~b} \overline{\mathrm{b}} \rightarrow \mu+X$ and minimum-bias events, it still gives about $10^{7}$ events on tape for each of these channels. Also the existence of supersymmetric particles, for example gluinos with $m_{\tilde{\mathrm{g}}} \approx 1 \mathrm{TeV} / c^{2}$, or a Higgs with $m_{\mathrm{H}} \approx 130 \mathrm{GeV} / c^{2}$, would result in sizeable events statistics $\left(10^{3}-10^{4}\right)$. Summing up everything, we estimate some $10^{7}$ events to tape every three days. Integrated over a full year, this amounts to 1 PByte of data per experiment. Apart the computing challenge to be faced for the data storage, distribution, reconstruction and analysis, this means that the statistical uncertainties will be negligible after a few days, for most of the physics cases. The analyses results will be dominated by systematic uncertainties, be it the detailed understanding of the detector response, theoretical uncertainties or the uncertainty from the luminosity measurements. A very detailed review of the early physics cases and the related analysis issues can be found in Ref. [18].

\subsection{Tests of the Standard Model}

There are many good reasons to investigate considerable efforts in the measurements of SM processes. We are sure that these have to be seen and thus they can serve as a proof for a working detector (a necessary requirement before any claim of discovery is made). Above we have mentioned that some SM processes are excellent tools to calibrate parts of the detector. However, such measurements are also interesting in their own right. We will be able to challenge the SM predictions at unprecedented energy and momentum transfer scales, by measuring cross sections and event features for minimum-bias events, QCD jet production, $\mathrm{W}$ and $\mathrm{Z}$ production with their leptonic decays, as well as top quark production. This will allow to check the validity of the Monte Carlo generators, both at the highest energy scales and at small momentum transfers, such as in models for the omnipresent underlying event. The parton distribution functions (pdfs) can be further constrained or measured for the first time in kinematic ranges not accessible at HERA. Important tools for pdf studies will be jet+photon production or Drell-Yan processes. Of course, SM processes are backgrounds for the new physics searches. In particular W/Z+jets, QCD multi-jet and top pair production will be important backgrounds to a large number of searches and therefore have to be understood in detail. In the following two examples are discussed a bit further.

Most likely the theoretically best known cross section at LHC will be for lepton pair production, via the Drell-Yan production of $\mathrm{W}$ and $\mathrm{Z}$ bosons. In 2004 the first differential next-to-nextto-leading order (NNLO) QCD calculation for vector boson production in hadron collisions was 

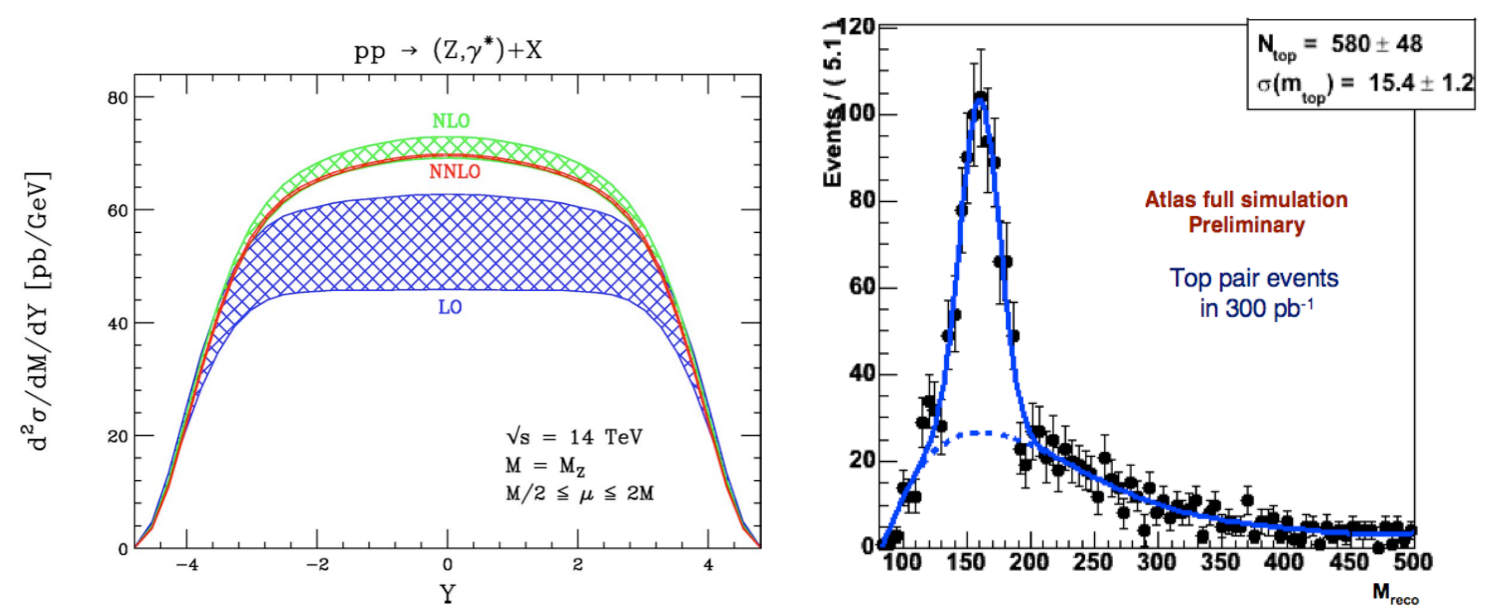

Figure 3: Left : Predictions for the rapidity dependence of $\mathrm{Z}$ boson production at LHC, at various orders in QCD perturbation theory [20]. Right : ATLAS simulation of the top quark mass reconstruction for an integrated luminosity of $300 \mathrm{pb}^{-1}$ [23].

completed by Anastasiou et al. [20]. This group has calculated the rapidity dependence for $\mathrm{W}$ and $\mathrm{Z}$ production at NNLO (Fig. 3, left). They have shown that the perturbative expansion stabilizes at this order in perturbation theory and that the renormalization and factorization scale uncertainties are drastically reduced, down to the level of one per-cent. Recent studies [21] conclude that the dominant uncertainties will be related to the knowledge of the pdfs, currently estimated at the 4-5\% level. This pdf uncertainty, as well as experimental and luminosity uncertainties, can be considerably reduced or completely eliminated by looking at ratios of cross sections, such as the rapidity dependence of $\mathrm{W}^{+} / \mathrm{W}^{-}$. On the other hand, the rapidity dependence of vector boson production will impose important constraints on the available pdf sets. Finally, in Ref. [22] it has been shown that the Drell-Yan process will be an important alternative tool for the determination of the machine and parton luminosities. Again, if normalized to the Drell-Yan production of, eg., $\mathrm{Z}$ bosons, many other SM processes can be predicted with considerably reduced uncertainties and without the knowledge of the machine luminosity.

LHC will be a top factory and thus offer a very rich top physics programme. Basically top quark production will be seen immediately. As a recent ATLAS study shows [23], even with a simple selection a very clear peak in the distribution of the reconstructed top mass is obtained with very small integrated luminosity (Fig. 3, right). The selection only requires missing transverse energy, one high- $p_{\mathrm{T}}$ and isolated lepton, at least four jets and a cut on the reconstructed hadronic $\mathrm{W}$ mass, but no b-tag. From this it is clear that, contrary to the TEVATRON, top production will be an important calibration tool, both for the jet energy scale by looking at the hadronically reconstructed $\mathrm{W}$ mass and for the b-tagging algorithms. Some of the physics topics to be addressed will be the measurements of top quark properties, such as its production and decay probabilities, its couplings, spin and mass. For the latter an ultimate precision of $1 \mathrm{GeV} / c^{2}$ is claimed to be achieved.

\subsection{Early discovery scenarios}

Regarding possible scenarios of discoveries to be made with the first year's LHC data, we 
summarize a study published in Ref. [18]. There three cases have been identified according to the experimental and theoretical difficulties to be faced in order to have an unambiguous claim of discovery : (a) An "easy" case would be the appearance of a new heavy resonance which decays into electron or muon pairs; (b) an intermediate case could be the search for supersymmetric particles; (c) the discovery of a very light Higgs $\left(m_{\mathrm{H}} \approx 120 \mathrm{GeV} / c^{2}\right)$ is considered to be a difficult case. Whatsoever scenario is realized in nature, it is clear that the LHC is a perfect place to look for evidence of new physics in the $\mathrm{TeV}$ energy range, thanks to the large phase space and the large rates for new particles production predicted by many of the SM extensions.

First we discuss the "easy" case. We use the notation Z' for any generic new heavy gauge boson with a mass up to several $\mathrm{TeV} / \mathrm{c}^{2}$. Such heavy gauge bosons appear in models with extensions of the SM gauge group, such as the recent Little Higgs models [6], in theories with dynamical electro-weak symmetry breaking or generally in Grand Unified Theories (GUTs) (see Ref. [24] for an overview of Z' searches). If such a heavy gauge boson has SM-like couplings to leptons and quarks, we could expect sizeable production cross sections and branching ratios to $\mathrm{e}^{+} \mathrm{e}^{-}$and $\mu^{+} \mu^{-}$, resulting in a very clear signature above a low and well understood background. The decay leptons would have very high transverse momentum and be isolated, thus easy to be triggered on. Figure 4 (left) shows the result of a CMS study [25] for the detection of a $Z_{\psi}$ [26] with a mass of 1 $\mathrm{TeV} / c^{2}$ via its decay into muon pairs, after trigger and offline reconstruction. Already with less than $1 \mathrm{fb}^{-1}$, such a signal cannot be missed. A similar study by ATLAS concludes that a Z', predicted by a Sequential SM [27], could be detected with about $1.5 \mathrm{fb}^{-1}$ up to masses of $2 \mathrm{TeV} / \mathrm{c}^{2}$, via its decay to $\mathrm{e}^{+} \mathrm{e}^{-}$. The necessary energy calibration and understanding of the lepton identification efficiency will be obtained from the processes $\mathrm{Z} \rightarrow \ell \ell+$ jet and Drell-Yan $\mathrm{Z}$ production, measured on the real data.

Heavy resonances also appear in models with extra dimensions. A particular realization of the Randall-Sundrum (RS) model with massive Kaluza-Klein excitations (gravitons) around $1 \mathrm{TeV}$ has been analyzed using the full detector simulation and reconstruction of CMS [28]. For sizeable couplings to electrons such a graviton would result in a very clear peak in the invariant mass distribution of high- $p_{\mathrm{T}}$ and isolated electron-positron pairs, over a very small background, allowing an unambiguous discovery with $10 \mathrm{fb}^{-1}$ of collected data. An important experimental aspect, which has been considered here, is that electrons in the TeV energy range may lead to saturation effects in the readout of the electromagnetic calorimeters. Once the existence of such a heavy resonance is established, it will be most exciting to analyse the further data and discriminate between the various models which predict such an object. Possible approaches will be to measure forward-backward asymmetries in the case of a Z' [29] or to look for other hints of extra dimensions such as large missing energy or very energetic isolated photons.

A large fraction of the analyses for an early discovery at LHC concentrate on supersymmetric extensions of the SM. Because of the extremely large parameter space, some specific benchmark models and points in parameter space have been chosen [31] for a more in-depth study of the discovery potential, such as mSUGRA with its minimal set of five parameters (for an introduction see [3] and references therein). Among these are the universal scalar $\left(m_{0}\right)$ and gaugino $\left(m_{1 / 2}\right)$ masses, which are fixed at the GUT scale. The full spectrum of supersymmetric particles at the TeV scale is then derived by simply employing the renormalization group equations. Over a considerable region of the parameter space, the production cross sections for supersymmetric partners of quarks 

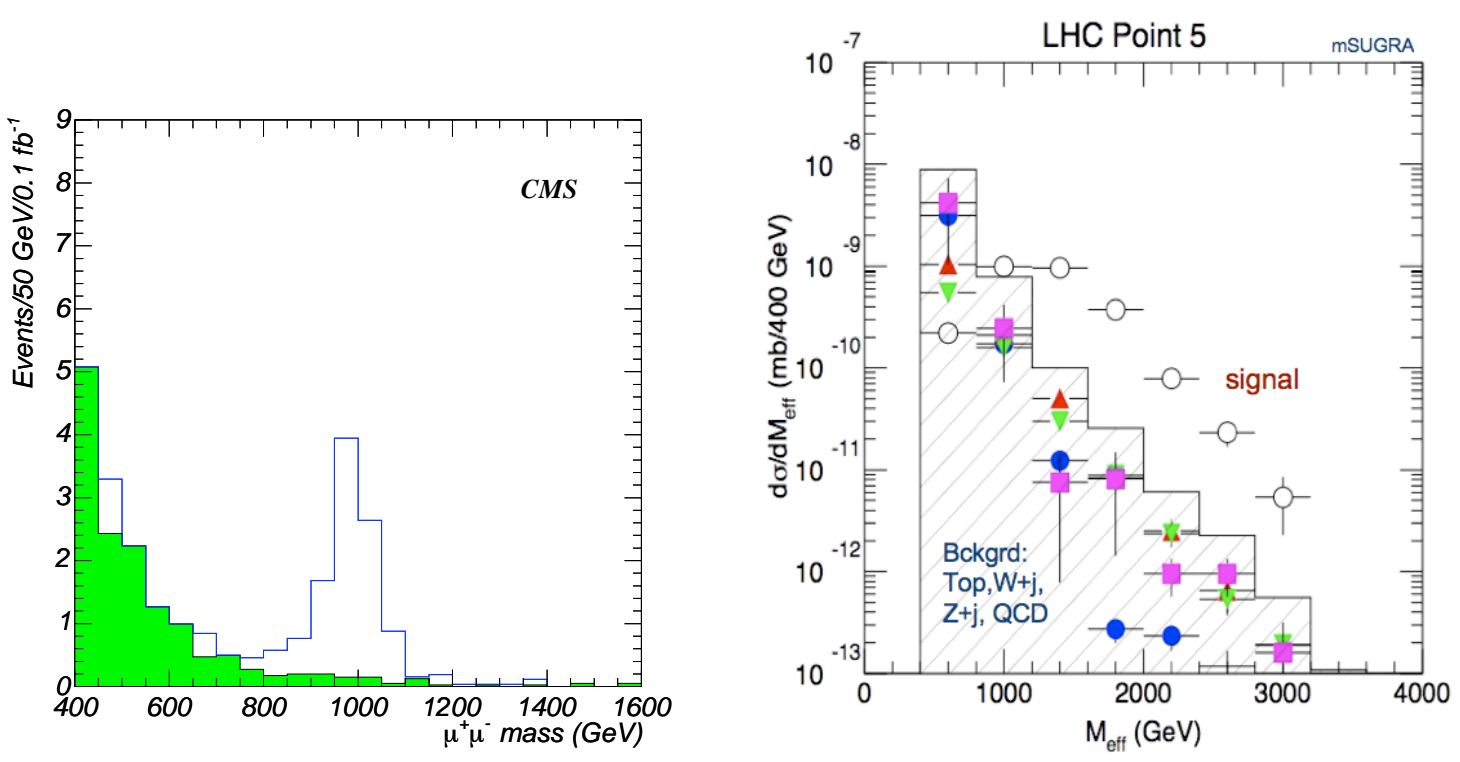

Figure 4: Left : Simulated appearance of a $Z_{\psi}$ resonance in the invariant mass spectrum of muon pairs measured in CMS [25]. Right : Reconstructed effective mass (see text for its definition) for various SM backgrounds and a SUSY signal as predicted for a particular point in the mSUGRA parameter space. The results have been obtained with a simulation of the ATLAS detector [30].

(squarks) and gluons (gluinos) are very large, thanks to their strong (QCD) couplings to the incoming partons. As an example, up to 100 events per day would be expected for gluino and squark masses of $\sim 1 \mathrm{TeV} / \mathrm{c}^{2}$, at a luminosity of $10^{33} \mathrm{~cm}^{-2} \mathrm{~s}^{-1}$. The subsequent decays, which typically occur via cascades to lighter supersymmetric (eg. charginos, neutralinos) and SM particles, lead to spectacular experimental signatures with very characteristic topologies. These are triggered on by looking for high-energetic multi-jet and multi-lepton events. If $R$-parity is conserved, the lightest SUSY particle (a neutralino in most models) would escape undetected. Therefore one of the most important characteristics (and trigger conditions) of such events would be large missing energy, $\mathbb{E}_{\mathrm{T}}$. A general approach of SUSY searches consists in the analysis of topological variables, such as the effective mass $M_{\text {eff }}=\mathbb{E}_{\mathrm{T}}+\sum p_{\mathrm{T}}^{\text {jet }}$, cf. Fig. 4 (right). A SUSY signal is expected to appear as an excess over the SM backgrounds (top production, W/Z+jets, QCD multi-jet events) in the large $M_{\text {eff }}$ region. However, in order to have clear evidence of a signal, two important aspects have to be considered. First, a good experimental understanding and calibration of the $\mathbb{E}_{\mathrm{T}}$ measurement is required. Second, a good theoretical control of the many SM backgrounds is not trivial to achieve with the current predictions and Monte Carlo models at hand. The problem is that multi-jet events, particularly in the high- $E_{\mathrm{T}}$ tail, are known to be badly simulated by the widely used parton shower models. The incorporation of matrix element corrections is absolutely essential for a reliable prediction [18]. Various approaches in this direction have appeared recently [32], but still a lot of effort is needed in order to reach a mature level of understanding. Therefore an early SUSY discovery via a topological search is considered to be of "intermediate" difficulty. However, if the abovementioned issues are under control, squarks with masses up to 1.5 (2) $\mathrm{TeV} / \mathrm{c}^{2}$ will be discovered with only $1(10) \mathrm{fb}^{-1}$ of data on tape. 
The electro-weak fits in the context of the SM, combined with the limits from the direct LEP searches, indicate that we can expect a Higgs boson with $114<m_{\mathrm{H}}<219 \mathrm{GeV} / c^{2}$ [33], with a preference for a very light Higgs, $m_{\mathrm{H}} \approx 120 \mathrm{GeV} / c^{2}$. In this mass range the dominant production mode is gluon fusion, but additional channels such as vector boson fusion (VBF) and associated Higgs production ( $\mathrm{WH}, \mathrm{ZH}, \mathrm{t} \overline{\mathrm{t}} \mathrm{H}$ ) are also considered. A very light Higgs dominantly decays to $\mathrm{b} \bar{b}$ pairs. Because of the enormously large QCD backgrounds, a detection in this decay channel might only be possible in the t⿱艹tH case. However, this will be extremely difficult, both because of experimental issues (jet energy scale, combinatorics, b-tagging) and the theoretical control of the backgrounds, such as $\mathrm{t}+$ jets. The channel $\mathrm{H} \rightarrow \tau \tau$ is investigated for VBF, which requires an excellent experimental understanding of very forward jets, a central jet veto and a $\tau$ trigger with good efficiency. Although their branching ratios are at the per-cent or even per-mille level, the channels $\mathrm{H} \rightarrow \mathrm{ZZ}^{*} \rightarrow 4 \ell(\ell=\mathrm{e}, \mu)$ and $\mathrm{H} \rightarrow \gamma \gamma$ are the most promising ones. In both cases the experimental signature would be a clear mass peak over a rather smooth background. In the former case good momentum resolution and understanding of the lepton isolation efficiency is required. The $\mathrm{H} \rightarrow \gamma \gamma$ case has been the benchmark channel for the design of the electromagnetic calorimeters, since a detection above the very large background is only possible with a mass resolution at the $1 \%$ level. This channel is studied for inclusive production, but also in the context of VBF and associated Higgs production. For a Higgs mass between 140 and $180 \mathrm{GeV} / c^{2}$, the most promising discovery channel turns out to be $\mathrm{H} \rightarrow \mathrm{WW}^{*} \rightarrow 2 \ell 2 v$, although a mass peak reconstruction is not possible because of the neutrinos. Nevertheless, with a jet veto and cuts related to the lepton kinematics a signal over background ratio above unity is achieved and a discovery should be in reach with the first few $\mathrm{fb}^{-1}$ of data [34].

In summary, in the context of the SM a Higgs with a mass around $120 \mathrm{GeV} / \mathrm{c}^{2}$ can be detected in the first $10 \mathrm{fb}^{-1}$ of data only if several channels (and the experiments) are combined. Each of the channels $\mathrm{H} \rightarrow \gamma \gamma, \overline{\mathrm{t}} \mathrm{H} \rightarrow \mathrm{b} \ell \nu$ bjj bb and qqH $\rightarrow \mathrm{qq} \tau \tau$ might contribute some $2 \sigma$ of total significance per experiment. Thus an observation of all channels is important. This requires well functioning experiments, due to the many different requirements on the detector performance, as well as a good theoretical understanding of the backgrounds ( $\mathrm{t} \overline{\mathrm{tj}}, \mathrm{t} \mathrm{t} j \mathrm{j}$, W/Z+jets, QCD multi-jets, prompt photon production) at the $10 \%$ level or better. It is worth noting that considerable progress has been made recently in the calculations of higher order QCD corrections and $p_{\mathrm{T}}^{\mathrm{H}}$ resummation for Higgs production, see eg. Refs. [35, 36]. Also the case of supersymmetric Higgs bosons is investigated, in particular for large $\tan \beta$ (the ratio of the vacuum expectation values of the two Higgs doublets) when the Higgs coupling to $b \bar{b}$ and $\tau^{+} \tau^{-}$is enhanced. Summaries of the discovery potentials for various SUSY parameter regions can be found in Refs. [37] and [38].

\subsection{The relevance of B-Physics}

Heavy flavour and in particular B-physics will be part of the toolkit to look for new physics at LHC. It will be possible to over-constrain the CKM matrix in many ways, to compare treelevel dominated processes to processes which involve penguin or box diagrams, and thus to reveal new (CP violating) physics in case discrepancies are found. The CKM-angle $\gamma$ will be measured by $\mathrm{LHCb}$ in various channels, with different sensitivity to new physics. For example, the timedependent $\mathrm{CP}$ asymmetry of $\mathrm{B}_{\mathrm{s}} \rightarrow \mathrm{D}_{\mathrm{s}}^{-} \mathrm{K}^{+}$depends on $\gamma$ already at the tree level, in contrast to $\mathrm{B}^{0} \rightarrow \pi^{+} \pi^{-}$and $\mathrm{B}_{\mathrm{s}} \rightarrow \mathrm{K}^{+} \mathrm{K}^{-}$. A comparison of the asymmetries in these channels, as well as of 
the various decay rates in the $\mathrm{B}^{0} \rightarrow \mathrm{D}^{0} \mathrm{~K}^{* 0}$ system will give further sensitivity. A special advantage of LHC compared to B-factories is the copious production of $\mathrm{B}_{\mathrm{s}}$ mesons. The $\mathrm{b} \rightarrow \mathrm{s}$ transition is an interesting candidate for the appearance of new physics. Rare decays could reveal new phenomena up to the TeV energy scale and complement the direct searches. Deviations from the SM predictions may be seen in the $\mathrm{B}_{\mathrm{s}} \rightarrow \phi \phi$ decay and in some regions of the SUSY parameter space the branching ratios for $\mathrm{B}_{\mathrm{s}} \rightarrow \mu^{+} \mu^{-}, \mathrm{B}_{\mathrm{d}} \rightarrow \mathrm{K}^{*} \mu^{+} \mu^{-}$and $\mathrm{B}_{\mathrm{s}} \rightarrow \phi \mu^{+} \mu^{-}$are considerably enhanced above the SM values. Finally, the $\mathrm{B}_{\mathrm{s}}$ mixing and oscillation may be affected. This could be observed by a larger than expected CP violation in $\mathrm{B}_{\mathrm{s}} \rightarrow \mathrm{J} / \psi \phi$ or by an oscillation frequency $\Delta m_{\mathrm{s}}$ which is higher than the SM expectation (around $20 \mathrm{ps}^{-1}$ ). The LHCb experiment is very well designed to measure the $\Delta m_{\mathrm{s}}$ parameter, thanks to its dedicated triggers, good particle identification capabilities and excellent proper time resolution (40 fs). This allows to achieve a $\Delta m_{\mathrm{s}}$ reach of $68 \mathrm{ps}^{-1}$ with one year of data taking. ATLAS and CMS do not have specialized particle identification, but will run at higher luminosities and thus complement LHCb in the analyses of rare decays. Reviews of the current status of B-physics and the LHCb physics programme can be found in Refs. [39] and [40].

\subsection{The study of heavy ion collisions}

The physics of heavy ions and strong phase transitions might enter a real discovery regime at LHC, thanks to the higher centre-of-mass energy and a ten-fold increase in the energy density $\varepsilon$ $\left[\mathrm{GeV} / \mathrm{fm}^{3}\right]$ compared to RHIC at Brookhaven. A particular feature of LHC is that for the first time high- $p_{\mathrm{T}}$ objects (jets, quarkonia) will be produced in heavy ion collisions, with huge statistics in a large variety of processes. Medium effects, such as medium-modified QCD radiation, are expected to be large and will be studied in detail. The energy loss of a high- $p_{\mathrm{T}}$ parton, when traversing the dense medium formed in heavy ion collisions, can be observed as jet quenching. At the same time it is interesting to measure the low- $p_{\mathrm{T}}$ tracks in order to get an understanding of the energy flow in these events. Jets, quarkonia and open flavour production are hard probes of the produced dense matter and will hopefully give unprecedented access to the equilibrium and non-equilibrium QCD dynamics. The LHC considerably extends the kinematic range in the small Bjorken- $x$ regime and therefore might give insight into phenomena such as perturbative saturation. Finally, collective phenomena such as radial and elliptic flow are expected to be even stronger than at RHIC, where indications have been reported that the formed medium shows strong collective behaviour, supporting the picture of an almost ideal liquid [41]. One of the first (immediate) measurements, already with about $10^{5}$ events, will be the determination of the actual multiplicity per unit rapidity. This rich physics programme will be addressed by ALICE and complemented by CMS and ATLAS. Qualitatively new experimental tools will be employed, such as very large rapidity coverage, high granularity and, in the special case of ALICE, excellent particle identification systems. A recent overview and outlook on heavy ion physics is found in Ref. [42].

\section{Physics reach and upgrades}

Obviously it is very difficult to predict the various physics scenarios after the first years of LHC running, since this strongly depends on early discoveries (or simple confirmations of the SM). If one or more Higgs bosons are found, then the next step will be to measure its (their) parameters (masses, couplings). However, in order to reach the ultimate precision and sensitivity, an integrated 
luminosity of up to $300 \mathrm{fb}^{-1}$ will be needed. Most likely the Higgs self-coupling will not be accessible, even with such a large data sample, and its measurement has to be postponed to a possible Super-LHC (SLHC). If there is first evidence for the production of SUSY particles, the following years will be devoted to the measurement of the sparticle masses, eg. by studying cascade decays and the related end-points of the lepton spectra. A non-trivial question to be answered will be the identification of the actually realized SUSY model and the underlying SUSY-breaking mechanism. Definitely one of the most outstanding successes of LHC would be the establishment of a clear connection to cosmological questions, eg. the discovery of a dark matter candidate such as a neutralino. Nevertheless, this has to be complemented by direct astro-physical observations in order to have a firm understanding of the important dark matter issue. At LHC it will be difficult or even impossible to observe sleptons with masses above $350 \mathrm{GeV} / c^{2}$, to explore the full gaugino mass spectrum, to measure the spin-parity and couplings of all sparticles and to disentangle squarks of the first two generations. An international linear collider would definitely offer a complementary approach to these problems. Finally, if none or only some of the above discoveries are made, all efforts will be dedicated to the search for other mechanisms of electro-weak symmetry breaking and/or extra dimensions. In the case no Higgs is found, we will concentrate on the resonant ('easy') or non-resonant ('very difficult') scattering of the longitudinal components of two W bosons, since unitarity conservation tells us that something has to happen there when approaching the TeV energy scale. Ultimately it could be that we have to accept fine tuning, as for example suggested by "Split SUSY" models [43]. Whatever nature is going to offer us, we are convinced that the LHC experiments are designed such that they are also ready for the unexpected. A more detailed discussion of the ultimate physics reach can be found in Refs. [8] and [9].

Possible upgrade scenarios of the machine and the experiments in view of SLHC are already under study. For the machine, the first step would be to push its parameters to the ultimate limits (eg. increase the beam energy to $7.45 \mathrm{TeV}$ and the intensity to $1.7 \times 10^{11}$ protons/bunch), without any hardware modifications. In a second phase the performance would be further improved by modifications in the insertion regions, for examples regarding the superconducting triplet quadrupole magnets. The bunch spacing may be reduced to $25 \mathrm{~ns}$ (if the electron cloud effect is under control), the $\beta^{*}$ lowered to $0.25 \mathrm{~m}$ and the beam crossing angle increased to $445 \mu \mathrm{rad}$. Although the peak luminosity might reach values close to $10^{35} \mathrm{~cm}^{-2} \mathrm{~s}^{-1}$, future studies will have to watch closely the luminosity lifetime (efficiency) of the machine, due to an increased energy stored in the beams and thus increased demands on machine control and protection. A further performance upgrade in a third phase would only be possible with major hardware modifications.

Also within the experiments the R\&D for a SLHC upgrade has started already now. The detector upgrades have to take into consideration increased radiation levels and pile-up noise. A reduced bunch spacing will require hardware modifications for the first-level triggers. The forward regions will be under particular radiation pressure, thus an improved shielding is under discussion. Generally, the developments go into the direction of faster and more granular sub-detectors, most notably new tracking systems. Recent reviews of the requirements and developments are found in Refs. [44] and [45]. 


\section{Conclusions}

A very exciting period for particle physics is lying ahead of us. At the LHC we will explore the $\mathrm{TeV}$ energy scale for the first time, with a direct discovery potential up to several $\mathrm{TeV}$. We expect to answer many of the current questions regarding the extensions of the SM, and possibly new questions will arise. CERN is fully committed to the LHC project. The machine and the detectors will be ready for first beams in summer 2007 .

\section{Acknowledgements}

I would like to thank the conference organizers for the invitation and my many colleagues from the LHC machine and detector groups for their help in preparing this review. I also thank D. Treille for his comments on the manuscript.

\section{References}

[1] P. Lefevre et al., CERN/AC/95-05.

[2] F. Englert, R. Brout, Phys. Rev. 13 (1964) 321; P.W.B. Higgs, Phys. Lett. 12 (1964) 132; T.W.B. Kibble, Phys. Rev. 13 (1964) 585.

[3] S.P. Martin, hep-ph/9709356.

[4] N. Arkani-Hamed, S. Dimopoulos and G. Dvali, Phys. Lett. B429 (1998) 263.

[5] L. Randall and R. Sundrum, Phys. Rev. Lett. 83 (1999) 3370.

[6] T. Han, H.E. Logan and L.-T. Wang, hep-ph/0506313.

[7] For a review see K.D. Lane, hep-ph/9605257.

[8] J.G. Branson et al., Eur. Phys. J. direct C4 (2002) N1 [hep-ph/0110021].

[9] F. Gianotti, Probing the hierarchy problem with the LHC, to appear in the proceedings of the XXII International Symposium on Lepton-Photon Interactions at High Energy, Uppsala, Sweden, July 2005.

[10] G. Rolandi, The LHC machine and experiments, to appear in the proceedings of the XXII International Symposium on Lepton-Photon Interactions at High Energy, Uppsala, Sweden, July 2005.

[11] http://1hc.web.cern.ch/lhc/.

[12] ATLAS Technical proposal, CERN/LHCC/94-43.

[13] CMS Technical proposal, CERN/LHCC/94-38.

[14] TOTEM Technical proposal, CERN/LHCC/99-7.

[15] LHCb Technical proposal, CERN/LHCC/98-4.

[16] ALICE Technical proposal, CERN/LHCC/95-71.

[17] J.M. Jimenez et al., CERN-LHC-PROJECT-REPORT-634.

[18] F. Gianotti and M.L. Mangano, hep-ph/0504221 .

[19] F. Cavallari, The CMS electromagnetic calorimeter, these proceedings. 
[20] C. Anastasiou, L. Dixon, K. Melnikov and F. Petriello, Phys. Rev. D69 (2004) 094008 [hep-ph/0312266].

[21] M. Dittmar et al., hep-ph/0511119.

[22] M. Dittmar, F. Pauss and D. Zurcher, Phys. Rev. D56 (1997) 7284 [hep-ex/97050 04].

[23] I. van Vulpen and W. Verkerke, ATLAS note in preparation.

[24] I. Golutvin, E. Rogalev, M. Savina and S. Shmatov, hep-ph/ 0502009.

[25] R. Cousins, J. Mumford and V. Valuev, [CMS Collaboration], CMS-CR-2004-050.

[26] D. London and J.L. Rosner, Phys. Rev. D34 (1986) 1530.

[27] G. Altarelli, B. Mele and M. Ruiz-Altaba, Z. Phys. C45 (1989) 109.

[28] C. Collard and M.C. Lemaire, Eur. Phys. J. C40N5 (2005) 15.

[29] M. Dittmar, A. Djouadi and A.-S. Nicollerat, Phys. Lett. B583 (2004) 111 [hep-ph/030 020 ].

[30] ATLAS Collaboration, ATLAS Detector and Physics Performance, Vol. 2, CERN/LHCC/99-15.

[31] M. Battaglia et al., Eur. Phys. J. C33 (2004) 273 [hep-ph / 0306219 ].

[32] For a review see M.A. Dobbs et al., hep-ph/0403045.

[33] M.W. Grünewald, Precision Tests of the Standard Model, these proceedings.

[34] M. Dittmar and H.K. Dreiner, Phys. Rev. D55 (1997) 167 [hep-ph/9608317].

[35] C. Anastasiou, K. Melnikov and F. Petriello, hep-ph/0501130.

[36] G. Bozzi, S. Catani, D. de Florian and M. Grazzini, hep-ph/0508068.

[37] S. Abdullin et al., Eur. Phys. J. C39S2 (2005) 41.

[38] M. Carena, S. Heinemeyer, C.E.M. Wagner and G. Weiglein, hep-ph/0511023.

[39] M.-H. Schune, CP Violation and Heavy Flavours, these proceedings.

[40] S. Barsuk (LHCb Collaboration), The LHCb experiment: Status and expected physics performance, proceedings of the Beauty 2005 - 10th International Conference on B-Physics at Hadron Machines, Assisi (Perugia), Italy, June 2005.

[41] T. Peitzmann, Jet production and high $p_{T}$ hadrons at RHIC, to appear in the proceedings of the Hadron Collider Physics Symposium 2005, Les Diablerets, Switzerland, July 2005.

[42] U. Wiedemann, Heavy Ions: Theoretical status and perspectives, to appear in the proceedings of the Hadron Collider Physics Symposium 2005, Les Diablerets, Switzerland, July 2005.

[43] N. Arkani-Hamed, S. Dimopoulos, G.F. Giudice and A. Romanino, Nucl. Phys. B709 (2005) 3 [hep-ph/0409232].

[44] F. Gianotti, Nucl. Phys. Proc. Suppl. 147 (2005) 23.

[45] D. Green, AIP Conf. Proc. 753 (2005) 437. 